\title{
Temporal dynamics in viral shedding and transmissibility of COVID-19
}

\author{
Xi He ${ }^{1,3}$, Eric H. Y. Lau ${ }^{2,3 凶}$, Peng Wu², Xilong Deng1, Jian Wang1, Xinxin Hao², Yiu Chung Lau², \\ Jessica Y. Wong', Yujuan Guan', Xinghua Tan', Xiaoneng Mo1, Yanqing Chen', Baolin Liao', \\ Weilie Chen', Fengyu Hu', Qing Zhang', Mingqiu Zhong1, Yanrong Wu', Lingzhai Zhao', \\ Fuchun Zhang ${ }^{1}$, Benjamin J. Cowling ${ }^{2,4}$, Fang Li ${ }^{1,4}$ and Gabriel M. Leung ${ }^{2,4}$
}

\begin{abstract}
We report temporal patterns of viral shedding in 94 patients with laboratory-confirmed COVID-19 and modeled COVID-19 infectiousness profiles from a separate sample of 77 infector-infectee transmission pairs. We observed the highest viral load in throat swabs at the time of symptom onset, and inferred that infectiousness peaked on or before symptom onset. We estimated that $44 \%$ ( $95 \%$ confidence interval, $30-57 \%$ ) of secondary cases were infected during the index cases' presymptomatic stage, in settings with substantial household clustering, active case finding and quarantine outside the home. Disease control measures should be adjusted to account for probable substantial presymptomatic transmission.
\end{abstract}

SARS-CoV-2, the causative agent of COVID-19, spreads efficiently, with a basic reproductive number of 2.2 to 2.5 determined in Wuhan ${ }^{1,2}$. The effectiveness of control measures depends on several key epidemiological parameters (Fig. 1a), including the serial interval (duration between symptom onsets of successive cases in a transmission chain) and the incubation period (time between infection and onset of symptoms). Variation between individuals and transmission chains is summarized by the incubation period distribution and the serial interval distribution, respectively. If the observed mean serial interval is shorter than the observed mean incubation period, this indicates that a significant portion of transmission may have occurred before infected persons have developed symptoms. Significant presymptomatic transmission would probably reduce the effectiveness of control measures that are initiated by symptom onset, such as isolation, contact tracing and enhanced hygiene or use of face masks for symptomatic persons.

SARS (severe acute respiratory syndrome) was notable, because infectiousness increased around 7-10 days after symptom onset ${ }^{3,4}$. Onward transmission can be substantially reduced by containment measures such as isolation and quarantine (Fig. 1a) ${ }^{5}$. In contrast, influenza is characterized by increased infectiousness shortly around or even before symptom onset ${ }^{6}$.

In this study, we compared clinical data on virus shedding with separate epidemiologic data on incubation periods and serial intervals between cases in transmission chains, to draw inferences on infectiousness profiles.

Among 94 patients with laboratory-confirmed COVID-19 admitted to Guangzhou Eighth People's Hospital, 47/94 (50\%) were male, the median age was 47 years and 61/93 (66\%) were moderately ill (with fever and/or respiratory symptoms and radiographic evidence of pneumonia), but none were classified as 'severe' or 'critical' on hospital admission (Supplementary Table 1).

A total of 414 throat swabs were collected from these 94 patients, from symptom onset up to 32 days after onset. We detected high viral loads soon after symptom onset, which then gradually decreased towards the detection limit at about day 21. There was no obvious difference in viral loads across sex, age groups and disease severity (Fig. 2).

Separately, based on 77 transmission pairs obtained from publicly available sources within and outside mainland China (Fig. 1b and Supplementary Table 2), the serial interval was estimated to have a mean of 5.8 days ( $95 \%$ confidence interval (CI), 4.8-6.8 days) and a median of 5.2 days ( $95 \%$ CI, 4.1-6.4 days) based on a fitted gamma distribution, with 7.6\% negative serial intervals (Fig. 1c). Assuming an incubation period distribution of mean 5.2 days from a separate study of early COVID-19 cases $^{1}$, we inferred that infectiousness started from 12.3 days (95\% CI, 5.9-17.0 days) before symptom onset and peaked at symptom onset ( $95 \%$ CI, $-0.9-0.9$ days) (Fig. 1c). We further observed that only $<0.1 \%$ of transmission would occur before 7 days, $1 \%$ of transmission would occur before 5 days and $9 \%$ of transmission would occur before 3 days prior to symptom onset. The estimated proportion of presymptomatic transmission (area under the curve) was $44 \%$ (95\% CI, 30-57\%). Infectiousness was estimated to decline quickly within 7 days. Viral load data were not used in the estimation but showed a similar monotonic decreasing pattern.

In sensitivity analysis, using the same estimating procedure but holding constant the start of infectiousness from 5, 8 and 11 days before symptom onset, infectiousness was shown to peak at 2 days before to 1 day after symptom onset, and the proportion of presymptomatic transmission ranged from $37 \%$ to $48 \%$ (Extended Data Fig. 1).

Finally, simulation showed that the proportion of short serial intervals (for example, $<2$ days) would be larger if infectiousness were assumed to start before symptom onset (Extended Data Fig. 2). Given the 7.6\% negative serial intervals estimated from the infector-infectee paired data, start of infectiousness at least 2 days before onset and peak infectiousness at 2 days before to 1 day after onset would be most consistent with this observed proportion (Extended Data Fig. 3).

Here, we used detailed information on the timing of symptom onsets in transmission pairs to infer the infectiousness profile of

${ }^{1}$ Guangzhou Eighth People's Hospital, Guangzhou Medical University, Guangzhou, China. ${ }^{2}$ World Health Organization Collaborating Centre for Infectious Disease Epidemiology and Control, School of Public Health, University of Hong Kong, Hong Kong, SAR, China. ${ }^{3}$ These authors contributed equally: Xi He, Eric H. Y. Lau. ${ }^{4}$ These authors jointly supervised this work: Benjamin J. Cowling, Fang Li, Gabriel M. Leung. ${ }^{\circledR}$ e-mail: ehylau@hku.hk 
a
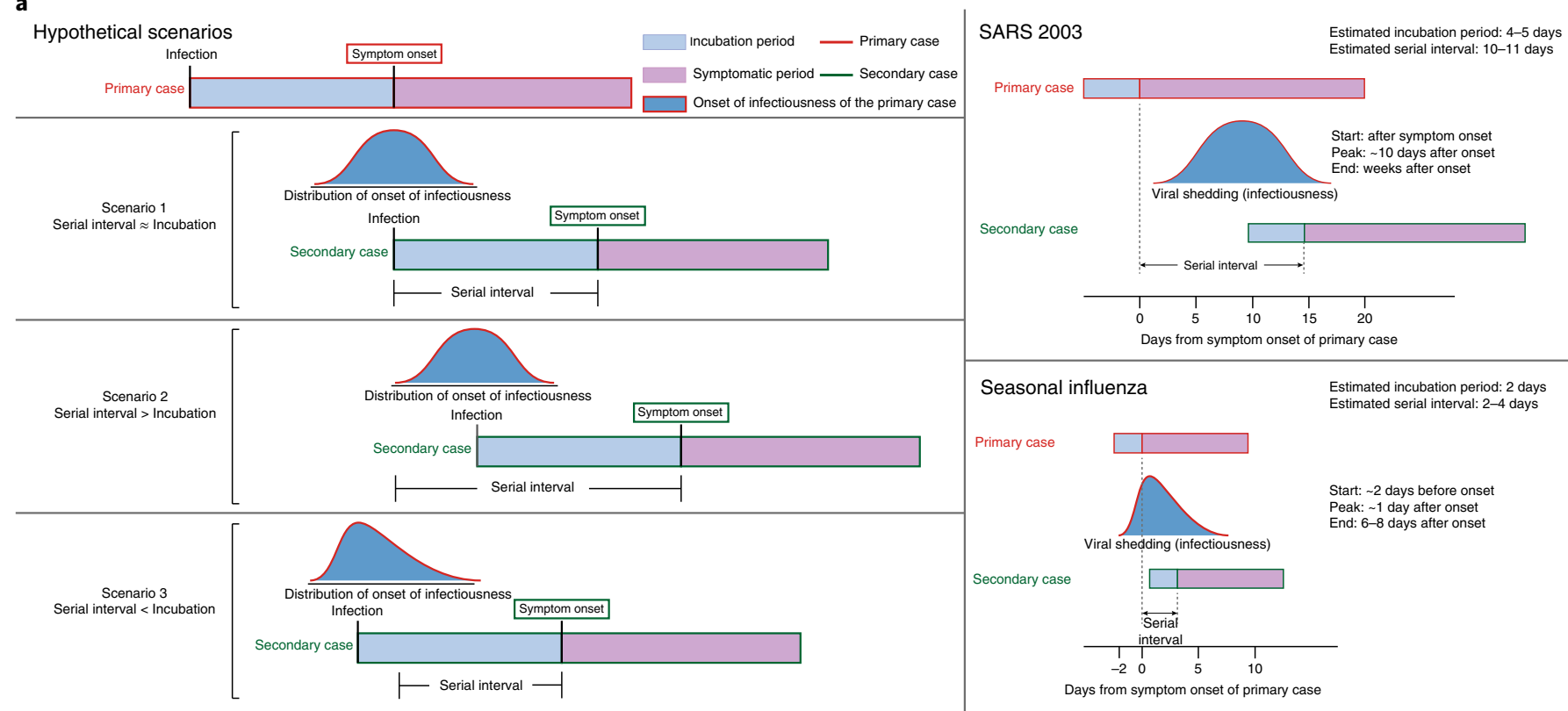

b
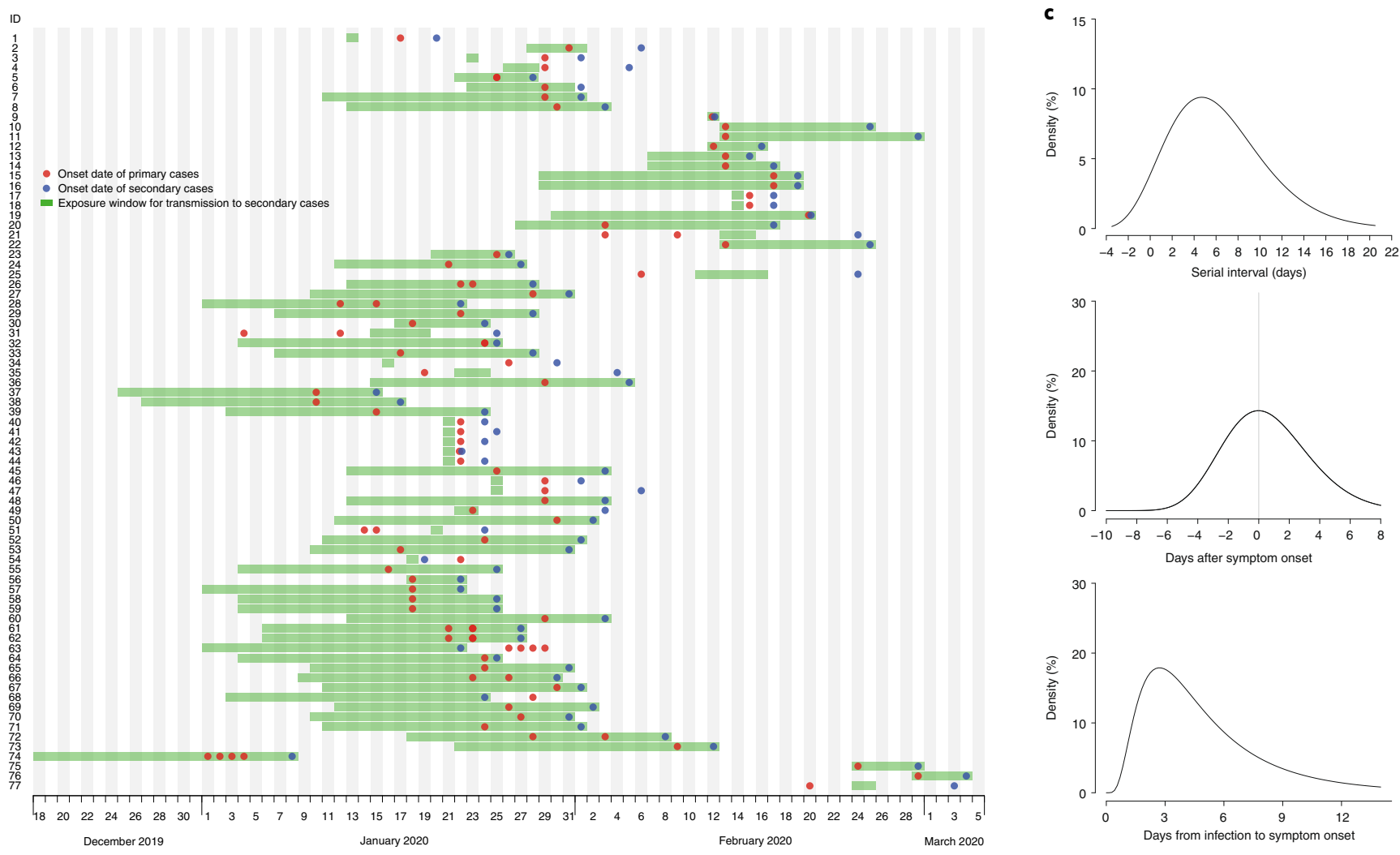

Fig. 1| Transmission of infectious diseases. a, Schematic of the relation between different time periods in the transmission of infectious disease.

b. Human-to-human transmission pairs of SAR-CoV-2 virus $(N=77)$. We assumed a maximum exposure window of 21 days prior to symptom onset of the secondary cases. Detailed information on the transmission pairs and the source of information is summarized in Supplementary Tables 2 and 3.

c, Estimated serial interval distribution (top), inferred infectiousness profile (middle) and assumed incubation period (bottom) of COVID-19.

COVID-19. We showed substantial transmission potential before symptom onset. Of note, most cases were isolated after symptom onset, preventing some post-symptomatic transmission. Even higher proportions of presymptomatic transmission of $48 \%$ and $62 \%$ have been estimated for Singapore and Tianjin, where active case finding was implemented ${ }^{7}$. Places with active case finding would tend to have a higher proportion of presymptomatic transmission, mainly due to quick quarantine of close contacts and isolation, thus reducing the probability of secondary spread later on in the course of illness. In a rapidly expanding epidemic wherein contact tracing/quarantine and perhaps even isolation are no longer feasible, or in locations where cases are not isolated outside the 

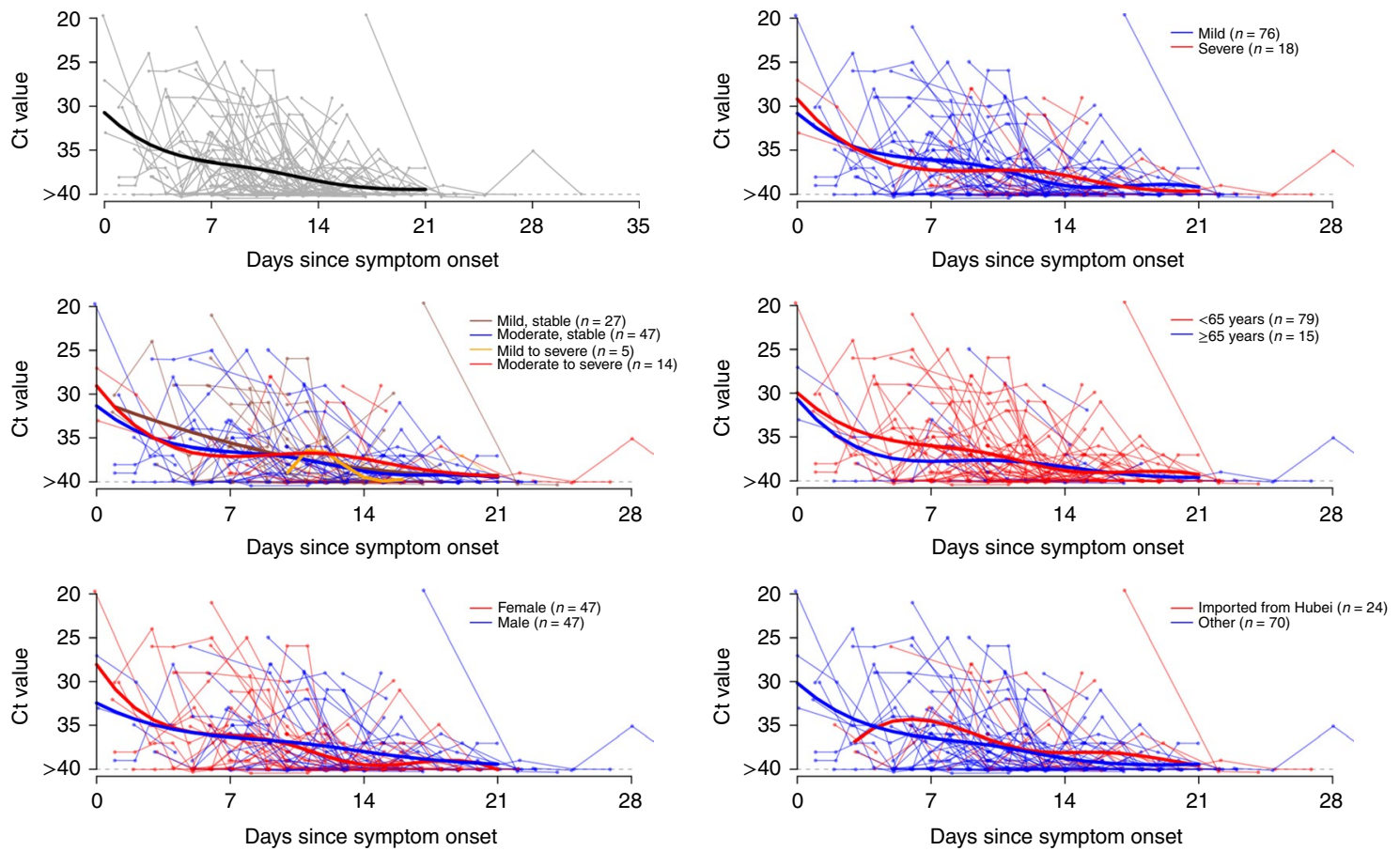

Fig. 2 | Temporal patterns of viral shedding. Viral load (threshold cycle (Ct) values) detected by RT-PCR (PCR with reverse transcription) in throat swabs from patients infected with SARS-CoV-2 $(N=94)$, overall and stratified by disease severity, sex, age group and link to Hubei province. The detection limit was $\mathrm{Ct}=40$, which was used to indicate negative samples. The thick lines show the trend in viral load, using smoothing splines. We added some noise to the data points to avoid overlaps.

home, we should therefore observe a lower proportion of presymptomatic transmission.

Our analysis suggests that viral shedding may begin 5 to 6 days before the appearance of the first symptoms. After symptom onset, viral loads decreased monotonically, consistent with two recent studies $^{8,9}$. Another study from Wuhan reported that virus was detected for a median of 20 days (up to 37 days among survivors) after symptom onset ${ }^{10}$, but infectiousness may decline significantly 8 days after symptom onset, as live virus could no longer be cultured (according to Wölfel and colleagues ${ }^{11}$ ). Together, these results support our findings that the infectiousness profile may more closely resemble that of influenza than of SARS (Fig. 1a), although we did not have data on viral shedding before symptom onset ${ }^{6,12}$. Our results are also supported by reports of asymptomatic and presymptomatic transmission ${ }^{13,14}$.

For a reproductive number of 2.5 ( ref. $^{2}$ ), contact tracing and isolation alone are less likely to be successful if more than $30 \%$ of transmission occurred before symptom onset, unless $>90 \%$ of the contacts can be traced ${ }^{15}$. This is more likely achievable if the definition of contacts covers 2 to 3 days prior to symptom onset of the index case, as has been done in Hong Kong and mainland China since late February. Even when the control strategy is shifting away from containment to mitigation, contact tracing would still be an important measure, such as when there are super-spreading events that may occur in high-risk settings including nursing homes or hospitals. With a substantial proportion of presymptomatic transmission, measures such as enhanced personal hygiene and social distancing for all would likely be the key instruments for community disease control.

Our study has several limitations. First, symptom onset relies on patient recall after confirmation of COVID-19. The potential recall bias would probably have tended toward the direction of under-ascertainment, that is, delay in recognizing first symptoms. As long as these biases did not differ systematically between infector and infectee, the serial interval estimate would not be substantially affected. However, the incubation period would have been overestimated, and thus the proportion of presymptomatic transmission artifactually inflated. Second, shorter serial intervals than those reported here have been reported, but such estimates lengthened when restricted to infector-infectee pairs with more certain transmission links ${ }^{16}$. Finally, the viral shedding dynamics were based on data for patients who received treatment according to nationally promulgated protocols, including combinations of antivirals, antibiotics, corticosteroids, immunomodulatory agents and Chinese medicine preparations, which could have modified the shedding dynamical patterns.

In conclusion, we have estimated that viral shedding of patients with laboratory-confirmed COVID-19 peaked on or before symptom onset, and a substantial proportion of transmission probably occurred before first symptoms in the index case. More inclusive criteria for contact tracing to capture potential transmission events 2 to 3 days before symptom onset should be urgently considered for effective control of the outbreak.

\section{Online content}

Any methods, additional references, Nature Research reporting summaries, source data, extended data, supplementary information, acknowledgements, peer review information; details of author contributions and competing interests; and statements of data and code availability are available at https://doi.org/10.1038/ s41591-020-0869-5.

Received: 15 March 2020; Accepted: 1 April 2020;

Published online: 15 April 2020

\section{References}

1. Li, Q. et al. Early transmission dynamics in Wuhan, China, of novel coronavirus-infected pneumonia. N. Engl. J. Med. 382, 1199-1207 (2020).

2. Wu, J. T., Leung, K. \& Leung, G. M. Nowcasting and forecasting the potential domestic and international spread of the 2019-nCoV outbreak originating in Wuhan, China: a modelling study. Lancet 395, 689-697 (2020). 
3. Peiris, J. S. et al. Clinical progression and viral load in a community outbreak of coronavirus-associated SARS pneumonia: a prospective study. Lancet 361, 1767-1772 (2003).

4. Pitzer, V. E., Leung, G. M. \& Lipsitch, M. Estimating variability in the transmission of severe acute respiratory syndrome to household contacts in Hong Kong, China. Am. J. Epidemiol. 166, 355-363 (2007).

5. Riley, S. et al. Transmission dynamics of the etiological agent of SARS in Hong Kong: impact of public health interventions. Science 300, 1961-1966 (2003)

6. Ip, D. K. et al. Viral shedding and transmission potential of asymptomatic and paucisymptomatic influenza virus infections in the community. Clin. Infect. Dis. 64, 736-742 (2017).

7. Ganyani, T. et al. Estimating the generation interval for COVID-19 based on symptom onset data. Preprint at medRxiv https://doi. org/10.1101/2020.03.05.20031815 (2020).

8. Zou, L. et al. SARS-CoV-2 viral load in upper respiratory specimens of infected patients. N. Engl. J. Med. 382, 1177-1179 (2020).

9. To, K.K.-W. et al. Temporal profiles of viral load in posterior oropharyngeal saliva samples and serum antibody responses during infection by SARS-CoV-2: an observational cohort study. Lancet Infect. Dis. https://doi. org/10.1016/S1473-3099(20)30196-1 (2020).
10. Zhou, F. et al. Clinical course and risk factors for mortality of adult inpatients with COVID-19 in Wuhan, China: a retrospective cohort study. Lancet 395, 1054-1062 (2020).

11. Wölfel, R. et al. Virological assessment of hospitalized patients with COVID-2019. Nature https://doi.org/10.1038/s41586-020-2196-x (2020).

12. Tsang, T. K. et al. Influenza A virus shedding and infectivity in households. J. Infect. Dis. 212, 1420-1428 (2015).

13. Bai, Y. et al. Presumed asymptomatic carrier transmission of COVID-19. JAMA https://doi.org/10.1001/jama.2020.2565 (2020).

14. Tong, Z. D. et al. Potential presymptomatic transmission of SARS-CoV-2, Zhejiang Province, China, 2020. Emerg. Infect. Dis.https://doi.org/10.3201/ eid2605.200198 (2020)

15. Hellewell, J. et al. Feasibility of controlling COVID-19 outbreaks by isolation of cases and contacts. Lancet Glob. Health 8, e488-e496 (2020).

16. Nishiura, H., Linton, N. M. \& Akhmetzhanov, A. R. Serial interval of novel coronavirus (COVID-19) infections. Int. J. Infect. Dis. 93, 284-286 (2020).

Publisher's note Springer Nature remains neutral with regard to jurisdictional claims in published maps and institutional affiliations.

๑) The Author(s), under exclusive licence to Springer Nature America, Inc. 2020 


\section{Methods}

Sources of data. Guangzhou Eighth People's Hospital in Guangdong, China was designated as one of the specialized hospitals for treating patients with COVID-19 at both city and provincial levels on 20 January 2020. After that, many people with COVID-19 were admitted via fever clinics, the hospital emergency room or after confirmation of cases from community epidemiological investigation carried out by the Guangzhou Center for Disease Control and Prevention, or transferred from other hospitals. The first confirmed patient with COVID-19 was admitted on 21 January 2020, but in the initial phase, patients suspected to have COVID-19 were also admitted. We identified all suspected and confirmed COVID-19 cases admitted from 21 January 2020 to 14 February 2020 and collected throat swabs in each case. Patients included those who traveled from Wuhan or Hubei to Guangzhou as well as locals, with cases ranging from asymptomatic, mild to moderate at admission.

The samples were tested by N-gene-specific quantitative RT-PCR assay as previously described ${ }^{17}$. To understand the temporal dynamics of viral shedding and exclude non-confirmed COVID-19 cases, we selected 94 patients who had at least one positive result (cycle threshold $(\mathrm{Ct})$ value $<40$ ) in their throat samples. Serial samples were collected from some but not all patients for clinical monitoring purposes.

We collected information reported on possible human-to-human transmission pairs of patients with laboratory-confirmed COVID-19 from publicly available sources, including announcements made by government health agencies and media reports in mainland China and countries/regions outside China. A transmission pair was defined as two confirmed COVID-19 cases identified in the epidemiologic investigation by showing a clear epidemiologic link with each other, such that one case (infectee) was highly likely to have been infected by the other (infector), by fulfilling the following criteria: (1) the infectee did not report a travel history to an area affected by COVID-19 or any contact with other confirmed or suspected COVID-19 cases except for the infector within 14 days before symptom onset; (2) the infector and infectee were not identified in a patient cluster where other COVID-19 cases had also been confirmed; and (3) the infector and infectee pair did not share a common source of exposure to a COVID-19 case or a place where there were COVID-19 case(s) reported. We excluded possible transmission pairs without a clear exposure history reported prior to symptom onset. Data of possible transmission pairs of COVID-19 were extracted, including age, sex, location, date of symptom onset, type or relationship between the pair cases and time of contact of the cases.

Statistical analysis. We analyzed two separate data sets-clinical and epidemiologic - to assess presymptomatic infectiousness. First, we assessed longitudinal viral shedding data from patients with laboratory-confirmed COVID19 starting from symptom onset, where viral shedding during the first few days after illness onset could be compared with the inferred infectiousness. Second, the serial intervals from clear transmission chains, combined with information on the incubation period distribution, were used to infer the infectiousness profile, as described in the following.

We present SARS-CoV-2 viral loads in the throat swabs of each patient by day of symptom onset. To aid visualization, a smoothing spline was fitted to the $\mathrm{Ct}$ values to summarize the overall trend. Specifically, a generalized additive model, $E(Y)=\beta_{0}+s(t)$, with an identity link was fitted, where $Y$ are the Ct values, $\beta_{0}$ is the intercept and $s(t)$ is a cubic spline evaluated at $t$ days after symptom onset. We also compared the viral load by disease severity, age, sex and travel history from Hubei.

We fitted a gamma distribution to the transmission pairs data to estimate the serial interval distribution. We used a published estimate of the incubation period distribution to infer infectiousness with respect to symptom onset from the first 425 patients with COVID-19 in Wuhan with detailed exposure history ${ }^{1}$. We considered that infected cases would become infectious at a certain time point before or after illness onset $\left(t_{\mathrm{s} 1}\right)$. Infectiousness - that is, transmission probability to a secondary case-would then increase until reaching its peak (Fig. 1). The transmission event would occur at time $t_{\mathrm{I}}$ with a probability described by the infectiousness profile $\beta_{c}\left(t_{\mathrm{I}}-t_{\mathrm{s} 1}\right)$ relative to the illness onset date, assuming a gamma distribution $\beta(t)$ with a time shift $c$ to allow for start of infectiousness $c$ days prior to symptom onset; that is, $\beta_{c}(t)=\beta(t+c)$. The secondary case would then show symptoms at time $t_{\mathrm{s} 2}$, after the incubation period that is assumed to follow a lognormal distribution $g\left(t_{\mathrm{s} 2}-t_{\mathrm{I}}\right)$. Hence the observed serial intervals distribution $f\left(t_{\mathrm{s} 2}-t_{\mathrm{s} 1}\right)$ would be the convolution between the infectiousness profile and incubation period distribution. We constructed a likelihood function based on the convolution, which was fitted to the observed serial intervals, allowing for the start of infectiousness around symptom onset and window of symptom onset $\left(t_{\mathrm{s} 11}, t_{\mathrm{s} 1 \mathrm{u}}\right)$, given by

$$
L\left(t_{\mathrm{S} 1 \mathrm{u}}, t_{\mathrm{S} 11}, t_{\mathrm{S} 2} \mid \theta\right)=\int_{t_{\mathrm{S} 11}}^{t_{\mathrm{S} 1 \mathrm{u}}} \int_{-\infty}^{t_{\mathrm{S} 2}} \beta_{c}\left(t_{\mathrm{I}}-t_{\mathrm{S} 1}\right) g\left(t_{\mathrm{S} 2}-t_{\mathrm{I}}\right) \mathrm{d} t_{\mathrm{I}} \mathrm{d} t_{\mathrm{S} 1}
$$

A normalization factor can be added to account for the uncertainty in the symptom-onset dates of the index cases. Assuming a uniform distribution, the likelihood would differ only by a multiplicative constant and give the same estimates.

Parameters $\theta$, including the gamma distribution parameters and the start of infectiousness, were estimated using maximum likelihood. The $95 \%$ CIs were obtained by bootstrapping with 1,000 replications. We also performed sensitivity analyses by fixing the start of infectiousness from days 5,8 and 11 before symptom onset and inferred the infectiousness profile.

As an additional check, we simulated the expected serial intervals assuming the same aforementioned incubation period but two different infectiousness profiles, where infectiousness started on the same day and from 2 days before symptom onset, respectively. A recent study isolated live infectious SARS-CoV-2 virus from patients with COVID-19 up to 8 days after symptom onset ${ }^{11}$, thus we assumed the same duration of infectiousness. We also assumed that infectiousness peaked on the day of symptom onset. The timing of transmission to secondary cases was simulated according to the infectiousness profile using a lognormal and exponential distribution, respectively, where the serial intervals were estimated as the sum of the onset to transmission interval and the incubation period. We drew random samples for the transmission time relative to symptom onset of the infector $T_{\mathrm{I}} \approx \beta_{c}(t)$, and also the incubation period $T_{\mathrm{inc}} \approx f(t)$, then the simulated serial interval was $T_{\mathrm{I}}+T_{\mathrm{inc}}$. We also performed simulation considering combinations of different infectiousness profiles, with start of infectiousness 7 days before to 3 days after symptom onset, and peak infectiousness also 7 days before to 3 days after symptom onset. We present the distribution of the serial intervals and proportion of negative serial intervals over 10,000 simulations.

All statistical analyses were conducted in $\mathrm{R}$ version 3.6.3 (R Development Core Team)

Ethics approval. Data collection and analysis were required by the National Health Commission of the People's Republic of China to be part of a continuing public health outbreak investigation.

Reporting Summary. Further information on research design is available in the Nature Research Reporting Summary linked to this article.

\section{Data availability}

Detailed transmission pairs data in this study are provided in the Supplementary Information and viral shedding data will be available upon request and approval by a data access committee. The data access committee comprises leadership of the Guangzhou Eighth People's Hospital and the Guangzhou Health Commission. There is no restriction to data access.

\section{Code availability}

We provided the code for generating Fig. 1c in the Supplementary Information and at https://github.com/ehylau/COVID-19. Other codes are available upon request to the corresponding author.

\section{References}

17. Chen, W. et al. Detectable 2019-nCoV viral RNA in blood is a strong indicator for the further clinical severity. Emerg. Microbes Infect. 9, 469-473 (2020).

\section{Acknowledgements}

This work was supported by Department of Science and Technology of Guangdong Province (project no. 2020B111108001, to F.L.) and a commissioned grant from the Health and Medical Research Fund from the Government of the Hong Kong Special Administrative Region (to B.J.C.).

\section{Author contributions}

X. He, E.H.Y.L., P.W., B.J.C., F.L. and G.M.L. conceived and designed the study. X. He, X.D., J.W., Y.G., X.T., X.M., Y.C. and B.L. were responsible for clinical care and collected all biomaterials. W.C. and F.H. carried out laboratory testing. Q.Z., M.Z. and Y.W. collected and collated linked clinical-epidemiologic data. L.Z., F.Z. and F.L. supervised and coordinated all aspects of the study at Guangzhou Eighth People's Hospital. P.W., X. Hao, Y.C.L. and J.Y.W. collected and verified all infector-infectee transmission data. E.H.Y.L., B.J.C. and G.M.L. wrote the first draft. All authors contributed to data interpretation, critical revision of the manuscript and approved the final version of the manuscript.

\section{Competing interests}

The authors declare no competing interests.

\section{Additional information}

Extended data is available for this paper at https://doi.org/10.1038/s41591-020-0869-5.

Supplementary information is available for this paper at https://doi.org/10.1038/ s41591-020-0869-5.

Correspondence and requests for materials should be addressed to E.H.Y.L.

Peer review information Alison Farrell and João Monteiro were the primary editors on this article and managed its editorial process and peer review in collaboration with the rest of the editorial team.

Reprints and permissions information is available at www.nature.com/reprints. 

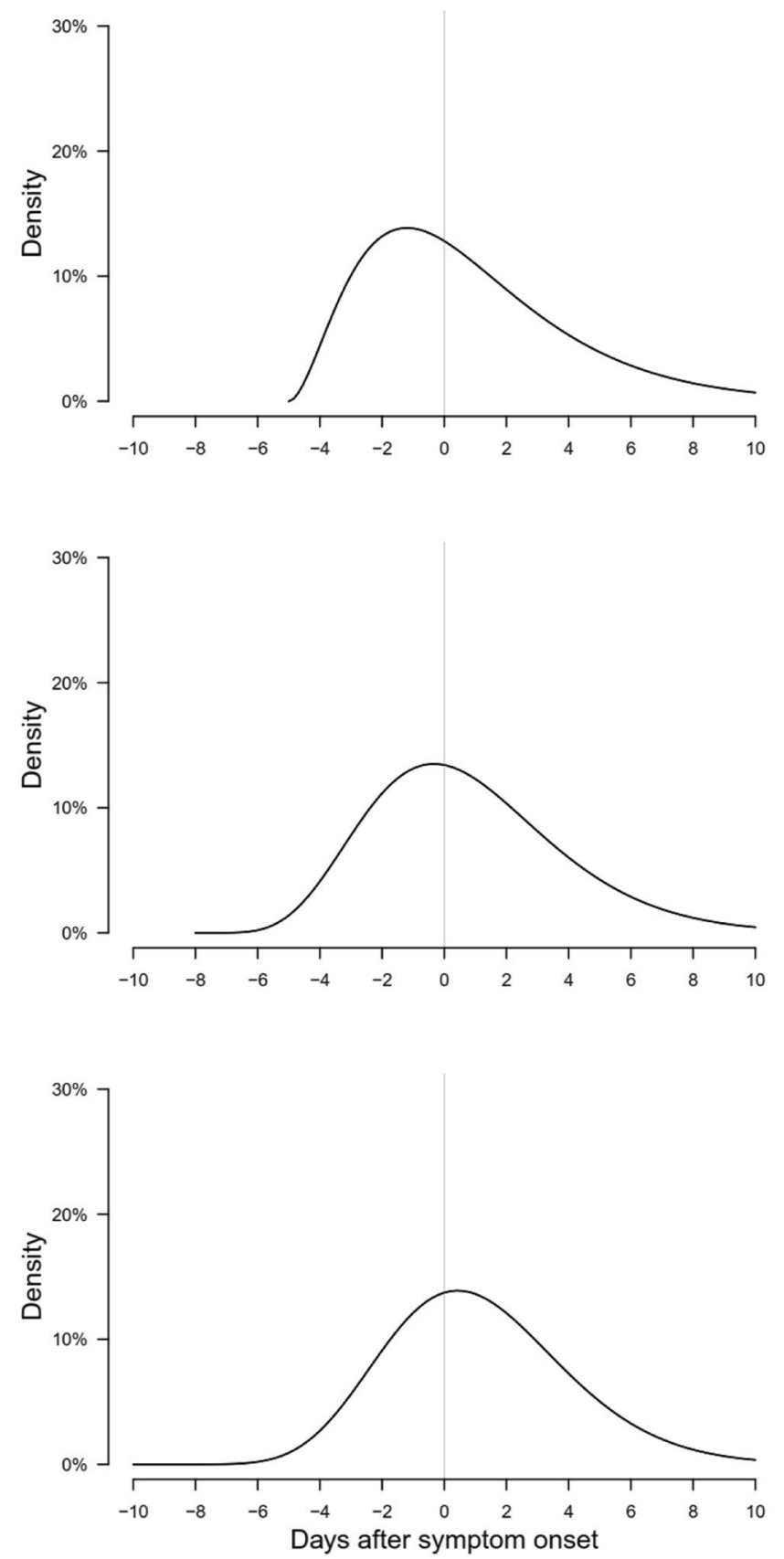

Extended Data Fig. 1 | Inferred infectiousness profile. Infectiousness was assumed to start from 5 days (top) to 8 days (middle) and 11 days (bottom) before symptom onset. 

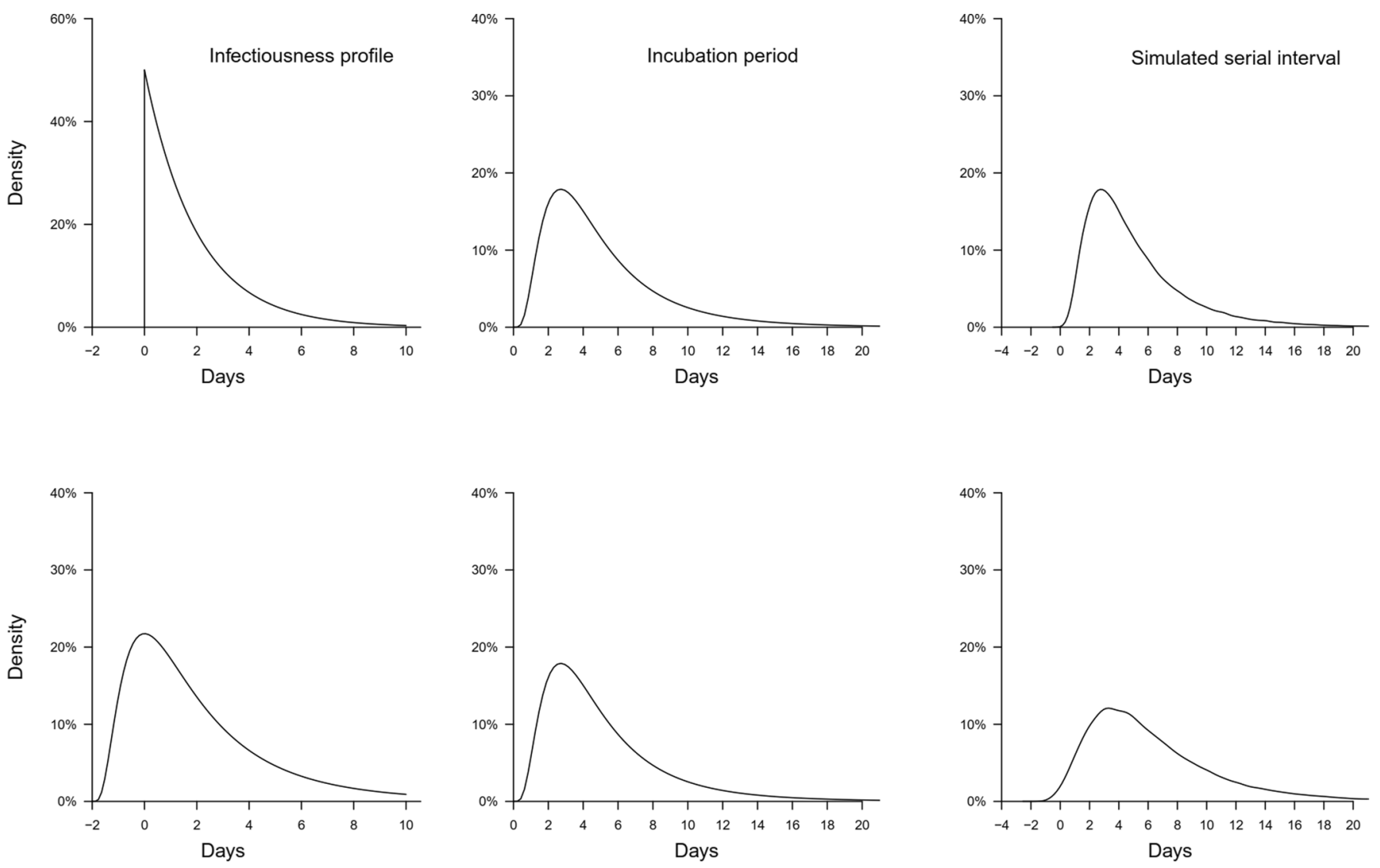

Extended Data Fig. 2 | Simulated serial intervals. Simulated serial intervals assuming infectiousness started on the same day of symptom onset (top panel) and from 2 days before symptom onset (bottom panel) to about 10 days after symptom onset. Both scenarios assumed that infectiousness peaked on the first day of symptom onset. 


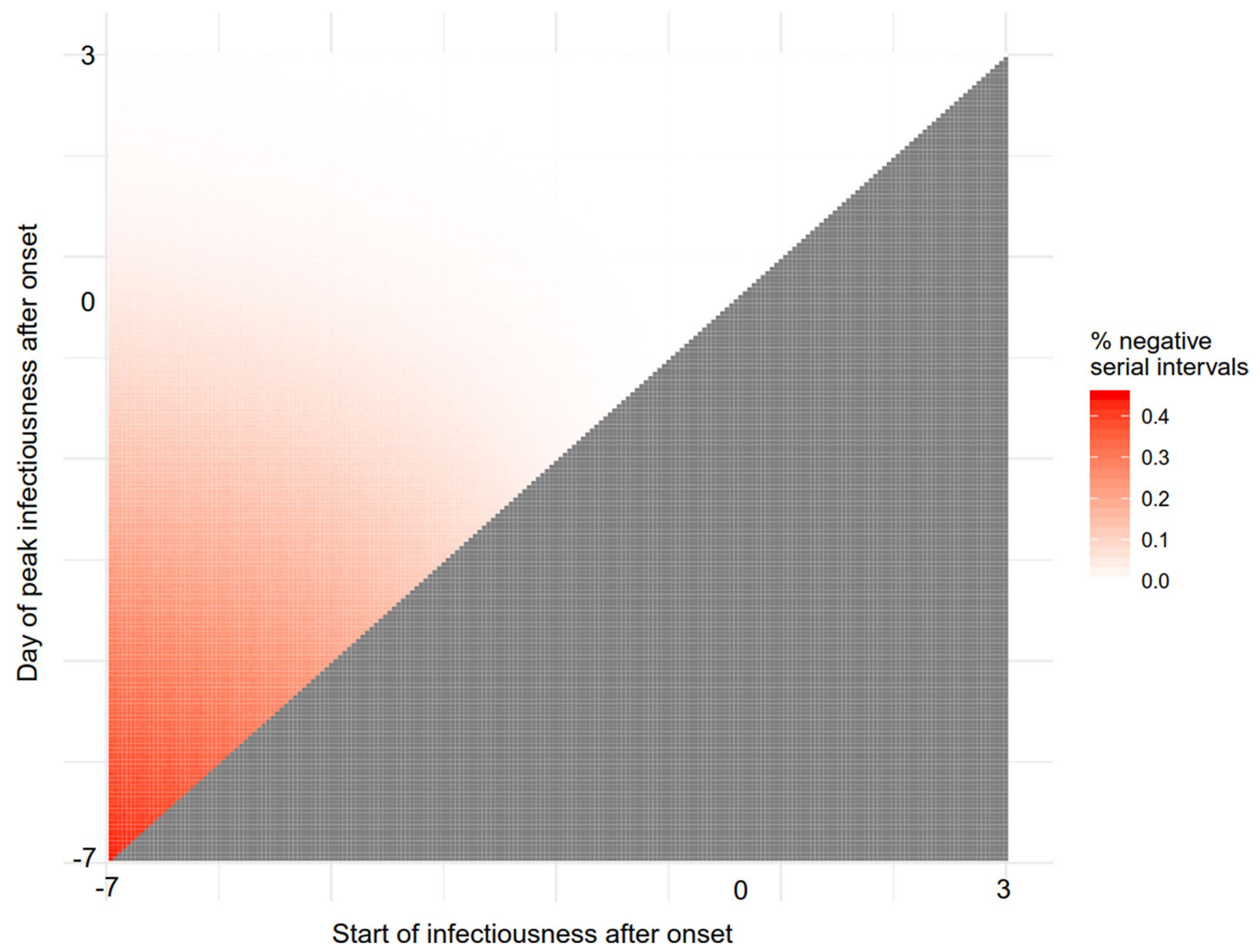

Extended Data Fig. 3 | Simulated proportions of negative serial intervals. Simulated proportions of negative serial intervals assuming start of infectiousness and peak infectiousness from 7 days before symptom onset to 3 days after symptom onset. From the estimated serial interval distribution based on infector-infectee pairs, $7.6 \%$ of the serial intervals were negative. Gray area represents the implausible range where the peak infectious is earlier than the start of infectiousness. 


\section{natureresearch}

Corresponding author(s): Eric HY Lau

Last updated by author(s): Mar 28, 2020

\section{Reporting Summary}

Nature Research wishes to improve the reproducibility of the work that we publish. This form provides structure for consistency and transparency in reporting. For further information on Nature Research policies, see Authors \& Referees and the Editorial Policy Checklist.

\section{Statistics}

For all statistical analyses, confirm that the following items are present in the figure legend, table legend, main text, or Methods section.

$\mathrm{n} / \mathrm{a}$ Confirmed

\ The exact sample size $(n)$ for each experimental group/condition, given as a discrete number and unit of measurement

$\square$ \ A statement on whether measurements were taken from distinct samples or whether the same sample was measured repeatedly

$\triangle \square$ The statistical test(s) used AND whether they are one- or two-sided

Xnly common tests should be described solely by name; describe more complex techniques in the Methods section.

$\bigotimes \square$ A description of all covariates tested

Х $\square$ A description of any assumptions or corrections, such as tests of normality and adjustment for multiple comparisons

A full description of the statistical parameters including central tendency (e.g. means) or other basic estimates (e.g. regression coefficient)

AND variation (e.g. standard deviation) or associated estimates of uncertainty (e.g. confidence intervals)

$\square$ For null hypothesis testing, the test statistic (e.g. $F, t, r$ ) with confidence intervals, effect sizes, degrees of freedom and $P$ value noted

Х Give $P$ values as exact values whenever suitable.

Х $\square$ For Bayesian analysis, information on the choice of priors and Markov chain Monte Carlo settings

Х $\square$ For hierarchical and complex designs, identification of the appropriate level for tests and full reporting of outcomes

$\bigotimes \square$ Estimates of effect sizes (e.g. Cohen's $d$, Pearson's $r$ ), indicating how they were calculated

Our web collection on statistics for biologists contains articles on many of the points above.

\section{Software and code}

Policy information about availability of computer code

Data collection MS Excel 2013.

Data analysis All statistical analyses were conducted in R version 3.6.2 (R Development Core Team, Vienna, Austria).

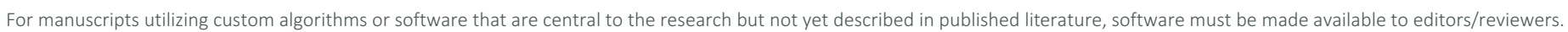
We strongly encourage code deposition in a community repository (e.g. GitHub). See the Nature Research guidelines for submitting code \& software for further information.

\section{Data}

Policy information about availability of data

All manuscripts must include a data availability statement. This statement should provide the following information, where applicable:

- Accession codes, unique identifiers, or web links for publicly available datasets

- A list of figures that have associated raw data

- A description of any restrictions on data availability

Detailed transmission pairs data in this study are given in the supplementary information and viral shedding data will be available upon request and approval by a data access committee. The data access committee comprises leadership of the Guangzhou Eighth People's Hospital and the Guangzhou Health Commission; there is no restriction to data access. 
Please select the one below that is the best fit for your research. If you are not sure, read the appropriate sections before making your selection. $\bigotimes$ Life sciences Behavioural \& social sciences Ecological, evolutionary \& environmental sciences

For a reference copy of the document with all sections, see nature.com/documents/nr-reporting-summary-flat.pdf

\section{Life sciences study design}

All studies must disclose on these points even when the disclosure is negative.

Sample size $\quad 94$ COVID-19 patients who had at least one positive results (Cycle threshold value < 40) by in their throat samples, tested by N-gene-specific quantitative reverse-transcriptase-polymerase-chain-reaction (RT-PCR) assay; 77 infector-infectee pairs from publicly available data

Data exclusions

No data was excluded

Replication

No replication

Randomization

Observational study, no randomization

Blinding

Observational study, no blinding

\section{Reporting for specific materials, systems and methods}

We require information from authors about some types of materials, experimental systems and methods used in many studies. Here, indicate whether each material, system or method listed is relevant to your study. If you are not sure if a list item applies to your research, read the appropriate section before selecting a response.

Materials \& experimental systems

$\mathrm{n} / \mathrm{a}$ Involved in the study

\ $\square$ Antibodies

Х Eukaryotic cell lines

\ $\square$ Palaeontology

$\bigotimes \square$ Animals and other organisms

$\square$ \uman research participants

\ $\square$ Clinical data

\begin{tabular}{l|l} 
Methods \\
\hline n/a & Involved in the study \\
$\triangle$ & $\square$ ChIP-seq \\
$X$ & $\square$ Flow cytometry \\
$\triangle$ & $\square$ MRI-based neuroimaging
\end{tabular}

\section{Human research participants}

Policy information about studies involving human research participants

Population characteristics

COVID-19 patients admitted to Guangzhou Eighth People's Hospital

Recruitment

Samples were collected from patients for clinical monitoring purposes.

Ethics oversight

Data collection and analysis were required by the National Health Commission of the People's Republic of China to be part of a continuing public health outbreak investigation.

Note that full information on the approval of the study protocol must also be provided in the manuscript. 